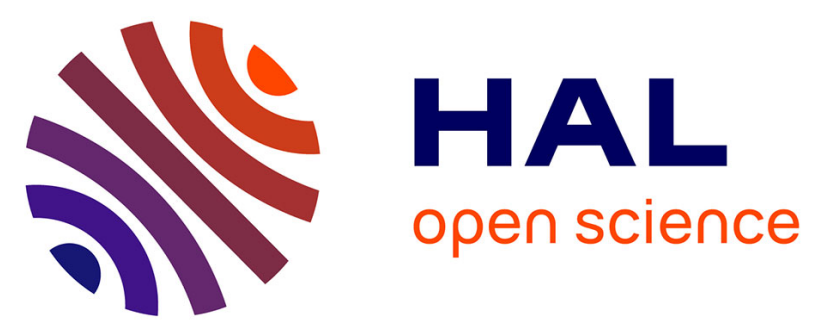

\title{
Le continuisme des mandats présidentiels, une dérive "caudilliste" du pouvoir exécutif? Réflexions à partir de la Constitution bolivienne de 2009
}

Victor Audubert

\section{- To cite this version:}

Victor Audubert. Le continuisme des mandats présidentiels, une dérive "caudilliste" du pouvoir exécutif? Réflexions à partir de la Constitution bolivienne de 2009. Revue française de droit constitutionnel, 2016, Nº108 (4), p. 751-768. 10.3917/rfdc.108.0751 . halshs-02482851

\section{HAL Id: halshs-02482851 \\ https://shs.hal.science/halshs-02482851}

Submitted on 3 Sep 2021

HAL is a multi-disciplinary open access archive for the deposit and dissemination of scientific research documents, whether they are published or not. The documents may come from teaching and research institutions in France or abroad, or from public or private research centers.
L'archive ouverte pluridisciplinaire HAL, est destinée au dépôt et à la diffusion de documents scientifiques de niveau recherche, publiés ou non, émanant des établissements d'enseignement et de recherche français ou étrangers, des laboratoires publics ou privés. 


\section{LE « CONTINUISME » DES MANDATS PRÉSIDENTIELS, UNE DÉRIVE « CAUDILLISTE » DU POUVOIR EXÉCUTIF ? RÉFLEXIONS À PARTIR DE LA CONSTITUTION BOLIVIENNE DE 2009}

\section{Victor Audubert}

Presses Universitaires de France | « Revue française de droit constitutionnel » 2016/4 N 108 | pages 751 à 768

ISSN 1151-2385

ISBN 9782130734246

Article disponible en ligne à l'adresse :

https://www.cairn.info/revue-francaise-de-droit-constitutionnel-2016-4-page-751.htm

Distribution électronique Cairn.info pour Presses Universitaires de France.

(C) Presses Universitaires de France. Tous droits réservés pour tous pays.

La reproduction ou représentation de cet article, notamment par photocopie, n'est autorisée que dans les limites des conditions générales d'utilisation du site ou, le cas échéant, des conditions générales de la licence souscrite par votre établissement. Toute autre reproduction ou représentation, en tout ou partie, sous quelque forme et de quelque manière que ce soit, est interdite sauf accord préalable et écrit de l'éditeur, en dehors des cas prévus par la législation en vigueur en France. Il est précisé que son stockage dans une base de données est également interdit. 
Le «continuisme » des mandats présidentiels, une dérive "caudilliste » du pouvoir exécutif? Réflexions à partir de la Constitution bolivienne de 2009

VICTOR AUDUBERT

«Dans le domaine de la politique, le Nouveau Monde s'est montré créateur, il a inventé le Président », affirmait André Siegfried ${ }^{1}$. C'est peu dire que l'Amérique latine a été féconde dans la mise en place de régimes politiques à "prédominance présidentielle ». Au travers des différentes chartes constitutionnelles qui ont jalonné l'histoire de la Bolivie, le pouvoir exécutif ${ }^{2}$ s'est vu structuré par un certain nombre de principes théoriques. Selon Jean-René Garcia, « on observe en Bolivie l'émergence d'une théorie du pouvoir d'État qui tend à compenser l'instabilité chronique par l'ancrage de principes constitutionnels d'autorité et de souveraineté3 $»$. On peut ainsi dégager la théorie du « pouvoir exécutif ambivalent ». Harvey Mansfield précise que « la beauté du pouvoir exécutif est donc d'être subordonné sans l'être, à la fois faible et fort. Il peut aller où la loi n'a pas sa place et pallier ainsi ses défauts tout en lui demeurant subordonné ${ }^{4} »$. Nous nous appuierons sur cette théorie pour démontrer que l'organe exécutif ${ }^{5}$ incarné par le président Evo Morales,

Victor Audubert, Doctorant contractuel et chargé de cours en droit public à l'université Paris-XIII, ses recherches portent sur le constitutionnalisme bolivien, et plus largement sur la philosophie du droit en Amérique latine. Victor Audubert est rattaché au Centre de recherche sur l'action locale (CERAL - EA 3968). victor.audubert@univ-paris13.fr

1. A. Siegfried, Amérique latine, Paris, A. Colin, 1934.

2. Selon le Lexique des termes juridiques Dalloz, le pouvoir exécutif peut se définir matériellement : «fonction consistant à assurer l'exécution des lois ». On a également une définition formelle : c'est un « organe (ou ensemble d'organes : chef d'État, cabinet ministériel) appelé aussi gouvernement, qui exerce la fonction exécutive ». Lexique des termes juridiques, Paris, Dalloz, $23^{\mathrm{e}}$ édition, 2015.

3. J.-R. Garcia, La Bolivie, histoire constitutionnelle et ambivalence du pouvoir exécutif, Paris, L'Harmattan, 2010, p. 14.

4. H. C. Mansfield Jr., Le Prince apprivoisé, de l'ambivalence du pouvoir, Paris, Fayard, 1994, p. 11.

5. La nouvelle CPE de 2009 a renommé les différents pouvoirs en " organes ». Ainsi, on trouve l'organe exécutif, l'organe législatif, l'organe judiciaire et l'organe électoral. Nous utiliserons ce terme uniquement pour désigner le pouvoir exécutif bolivien dans son organisation, au sens organique du terme, contrairement à la notion de "pouvoir ", qui porte ici un sens plus général et théorique. 
se trouve aujourd'hui dans une position ambivalente : à la fois obligé de collaborer avec différents pouvoirs politiques, mais également doté d'une légitimité et d'un pouvoir sans précédent dans un contexte de centralisation inédite du pouvoir.

La Bolivie, qui s'est longtemps caractérisée par son « instabilité politique » chronique ${ }^{6}$, connait une stabilité du pouvoir politique relative depuis une dizaine d'années. Après d'importants mouvements sociaux et indigénistes au début des années 2000, Evo Morales Ayma a été élu dès le premier tour à la fonction présidentielle en décembre 2005, inaugurant une nouvelle période de la vie politique bolivienne. Avec la réélection d'Evo Morales en 2009 et 2014, l'exécutif du nouvel «État plurinational » s'est révélé étonnamment stable - du moins en apparence. Si cette période de stabilité contraste évidemment avec les «présidences éphémères »-voire « éclairs »-qu'a connues la Bolivie depuis son indépendance en 1825, les fortes tensions sociales et «éco-territoriales » qui traversent la société bolivienne laissent planer un doute sur la stabilité effective du gouvernement bolivien.

Cette nouvelle Constitution politique de l'État (CPE), adoptée le 25 janvier 2009 par 61,43\% des votants et avec une participation de $90,26 \%$ des inscrits, est promulguée le 7 février 2009, et constitue un fait majeur dans l'histoire constitutionnelle de la Bolivie. Longue de 411 articles, la CPE redéfinit l'État bolivien, et réorganise les institutions politiques et judiciaires de la Bolivie. La première partie de la Constitution, avec 144 articles, renvoie aux principes fondamentaux de l'État, aux droits humains et aux nouveaux droits collectifs issus de la quatrième génération des droits. La deuxième partie a trait à l'organisation de l'État bolivien; la troisième quant à elle aborde la structure territoriale et les autonomies. La quatrième partie renvoie aux questions économiques, et la cinquième se consacre à la révision de la Constitution et à la place de cette dernière dans la hiérarchie des normes.

La nouvelle Constitution bolivienne porte un régime politique de type présidentiel s'inscrivant dans la tradition constitutionnelle bolivienne, avec un " Président de l'État ${ }^{\text {} ~}$ » élu au suffrage universel et une séparation relativement rigide des différents pouvoirs exécutif, législatif, judiciaire et électoral ${ }^{8}$. Bien que cette constitution trouve ses origines dans la nécessité de renouveler le pacte social bolivien qui s'était trouvé mis à mal au début des années 2000, les innovations théoriques et institutionnelles sont nombreuses. Ainsi, cette constitution a modifié la structure du pouvoir

6. Voir à ce sujet: J.-P. Lavaud, L'instabilité politique en Amérique latine. Le cas de la Bolivie, Paris, L'Harmattan, 1995.

7. La République étant abolie, il y a désormais en Bolivie un Président ou une Présidente de l'État.

8. Le pouvoir électoral constitue le quatrième pouvoir politique en Bolivie, et plus généralement dans le constitutionnalisme latino-américain. 
exécutif ${ }^{9}$, faisant évoluer la notion de régime présidentiel. Cependant, l'échec relatif de l'Assemblée constituante, la reprise en main du processus constituant par le pouvoir exécutif et le Congrès bolivien, et surtout la figure charismatique d'Evo Morales ont accentué la personnalisation du pouvoir ainsi que « le centralisme des décisions ${ }^{10}$ ». Cette résurgence d'une pratique « caudilliste » du pouvoir exécutif se caractérise notamment par une remise en cause du principe de limitation à une réélection du mandat présidentiel, principe pourtant au cœur du constitutionnalisme bolivien. A travers une interprétation controversée de la Constitution par le juge constitutionnel bolivien, et une tentative de modification de ce même texte en février 2016 via une procédure législative que l'on peut considérer à bien des égards comme inconstitutionnelle, le pouvoir exécutif bolivien participe d'une logique de «déconstitutionnalisation » en remettant en cause une constitution qu'il a lui-même œuvré à promulguer.

On se demandera donc dans quelle mesure ce «continuisme » des mandats présidentiels s'inscrit dans une pratique « caudilliste » du pouvoir exécutif bolivien. On verra que cette limitation des mandats, bien qu'elle soit au cœur du constitutionnalisme bolivien et du processus constituant de 2006 (I), est remise en cause par un pouvoir exécutif aux tentations de plus en plus « caudilliste », au profit d'un « continuisme » des mandats présidentiels (II).

\section{I - UNE LIMITATION DES MANDATS AU FONDEMENT DU PROCESSUS CONSTITUANT BOLIVIEN}

La Constitution bolivienne, si elle s’inscrit dans la tradition présidentielle propre au constitutionnalisme latino-américain, refonde dans une certaine mesure le pouvoir exécutif et ses relations avec les autres pouvoirs politiques. On peut parler à ce sujet d'un «présidentialisme

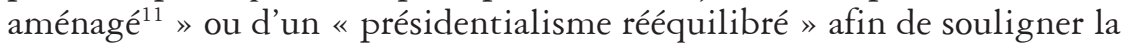
volonté du constituant bolivien de limiter les pouvoirs de l'exécutif, en particulier avec le principe de limitation des mandats présidentiels (A). Cependant, la pratique est marquée par une concentration inédite des pouvoirs au sein de l'organe exécutif, mettant ainsi à mal le fragile équilibre des pouvoirs politiques (B).

9. Selon l'article 165 de la CPE, l'organe exécutif est composé par « la Président ou le Président, la Vice-Présidente ou le Vice-Président, les Ministres de l'État ».

10. J. Costa Benavides, «Los cambios en la estructura y organización del Poder Ejecutivo ante la nueva estructura y organización territorial del Estado de la nueva Constitución », Reflexión crítica sobre la nueva CPE, La Paz, Fundación Konrad Adenauer, 2009, p. 270.

11. V. Souty, «La nouvelle Constitution politique de l'État bolivienne », Revue française de droit constitutionnel, 2011, $\mathrm{n}^{\circ} 85$, pp. 203-224. 


\section{A - UN RÉGIME PRÉSIDENTIEL « RÉÉQUILIBRÉ » PORTÉ PAR LA CONSTITUTION DE 2009}

Ce principe de limitation des mandats se trouve au cœur du constitutionnalisme bolivien (1), et notamment dans la Constitution bolivienne de 2009 où il est cependant aménagé (2).

1 - Le principe de non-renouvellement au fondement du constitutionnalisme bolivien

Depuis la vague d'indépendance du début du XIX siècle, les régimes politiques latino-américains - la très grande majorité, si ce n'est la totalité, d'inclination présidentielle - se sont employés à limiter le cumul des mandats électoraux dans le temps, notamment en encadrant et limitant la réélection présidentielle immédiate.

Dans l'histoire constitutionnelle de la Bolivie, le principe de la nonréélection pour le mandat présidentiel fut appliqué de manière aléatoire. Cela allait de la prohibition pure et simple à l'autorisation la plus libérale, en passant par l'autorisation conditionnée. Ainsi, dans la Constitution bolivarienne de 1826 , on trouve une présidence à vie ${ }^{12}$, taillée pour le père de l'indépendance latino-américaine Simon Bolivar. Par la suite, la Constitution de 1831 fut le seul texte à autoriser la réélection présidentielle sans limitation. Les Constitutions de 1868 et 1961 autorisaient quant à elles la réélection immédiate. Tous les autres textes constitutionnels ${ }^{13}$, ceux de 1839, 1843, 1851, 1861, 1871, 1880, 1931, 1938, 1945, 1947, 1967, 1994 et 2004 ${ }^{14}$, interdisaient la réélection présidentielle immédiate, et l'autorisaient après une certaine période - généralement un mandat.

\section{2 - Un principe aménagé dans la Constitution de 2009}

Avec la Constitution de 2009, l'interdiction d'une réélection présidentielle immédiate est abandonnée avec l'article 168: «La période du mandat de la Présidente ou du Président et de la Vice-présidente ou du vice-président de l'Etat est de cinq ans, et ils peuvent être réélus une seule fois de manière continue. » Cette limitation du cumul du mandat présidentiel dans le temps est le fruit d'un accord politique entre le MAS et l'opposition de droite. En effet, lors du processus constituant, l'Assemblée constituante s'est retrouvée bloquée, ne parvenant pas à réunir les deux tiers des voix nécessaires à l'approbation du projet de texte

12. La "presidencia vitalista ", c'est-à-dire la présidence à vie en français.

13. Au nombre de 12 ou 14, selon que l'on considère que les révisions de 1994 et de 2004 de la Constitution de 1967 débouchent - ou non - sur des nouvelles constitutions.

14. La Constitution de 2004, dans son article 87, interdisait la réélection immédiate du président ou du vice-président. 
constitutionnel. Dans ce contexte tendu, un accord politique fut trouvé entre le MAS et l'opposition de droite à travers la modification du projet de constitution lors du passage devant le Congrès national bolivien. Cet accord politique comportait une modification discrète, mais néanmoins capitale, avec l'ajout d'une Disposition Transitoire Première dans le texte constitutionnel ; celle-ci disposait, dans son second alinéa ${ }^{15}$, que les mandats antérieurs à la présente Constitution seraient pris en compte dans le décompte des nouveaux mandats, ce qui impliquait le renoncement du Président et du Vice-président actuels à une réélection en 2014.

Cette disposition constituait un gage pour l'opposition de droite : Evo Morales et Álvaro García Linera pourraient se présenter à leur réélection en 2009 - comme le prévoyait l'article 168 de la Constitution de 2009-, mais pas en 2014 pour un troisième mandat ${ }^{16}$. En échange de ce renoncement à se représenter en 2014, l'opposition acceptait de voter la nouvelle CPE au Congrès national. Evo Morales put donc se présenter en 2009, conformément à la Disposition transitoire. Cette disposition fut d'ailleurs reconnue juridiquement, avec la loi $\mathrm{n}^{\circ} 4021$ du Régime électoral transitoire, qui déclarait se fonder dans la disposition transitoire et qu'en application de ladite disposition, le premier mandat comptabilisé était celui qui était en ouvre lors de la promulgation de la nouvelle CPE, c'est-à-dire le premier mandat d'Evo Morales (2005-2009).

\section{B - UN POUVOIR POLITIQUE CONCENTRÉ AUX MAINS DE L'ORGANE EXÉCUTIF}

Avec une Assemblée constituante sous le contrôle des pouvoirs constitués, la forte popularité d'Evo Morales ainsi que l'émergence rapide du fait majoritaire en Bolivie, on constate que le texte constitutionnel était vicié avant même sa promulgation en faisant du pouvoir exécutif le seul centre de décision effectif en Bolivie (1). Par ailleurs, l'ajout d'un second tour à l'élection présidentielle tend à personnaliser un peu plus la vie politique bolivienne (2).

\section{1 - Une Constitution viciée avant même sa promulgation}

Alors que l'objectif premier de la nouvelle CPE était d'élargir la participation et le contrôle des citoyens, la fonction exécutive s'est trouvée renforcée en pratique. À travers une légitimité retrouvée, et en dépit de l'existence de contre-pouvoirs divers, le Président concentre de fait un

15. « Los mandatos anteriores a la vigencia de esta Constitución serán tomados en cuenta a los efectos del cómputo de los nuevos periodos de funciones ».

16. À ce sujet, Evo Morales avait promis que la Constitution de 2009 ne serait pas révisée «avant 50 ans ». 
pouvoir conséquent, en étant à la fois chef d'État et chef du gouvernement. On s'aperçoit par ailleurs que les attributions du chef de l'État ${ }^{17}$ sont beaucoup plus importantes que dans la Constitution de 1967 révisée en 2004. Ainsi, dans la Constitution de 1967 le Président « concourrait à la formulation des codes et des lois à travers des messages spéciaux »; de cette manière, la Constitution laissait au Congrès la tâche de formuler les lois selon les coalitions partisanes du moment. Dans la nouvelle CPE, le Président " propose et dirige les politiques du gouvernement et de l'État ». Il se substitue au législateur en déterminant la politique de la Nation, à la manière de l'exécutif français ${ }^{18}$. Par ailleurs, il est apte à « présenter des projets de loi d'urgence économique à l'ALP, qui devra les étudier en priorité ».

Par ailleurs, on note un certain contrôle politique sur la justice. Ainsi, les membres du Tribunal Suprême de Justice et du TCP peuvent être mis en accusation par la Chambre des députés, et jugés par le Sénat, la sentence étant adoptée à la majorité qualifiée des deux tiers ${ }^{19}$. Cette subordination de la justice au pouvoir législatif est évidemment dommageable, car elle entache la séparation - ou tout du moins l'équilibre - des pouvoirs et jette un doute sur la neutralité nécessaire des membres des deux instances les plus importantes du système judiciaire bolivien.

Afin d'éviter les situations de blocages inhérents aux régimes présidentiels, et en particulier au constitutionnalisme bolivien ${ }^{20}$, l'une des innovations majeures réside dans le rôle du peuple comme mécanisme de déblocage en cas de conflits entre l'exécutif et le législatif. De manière paradoxale, on a constaté ces dernières années que les contre-pouvoirs prévus par la CPE de 2009 n'étaient pas opérants, et que le principal contre-pouvoir au pouvoir exécutif était une partie de l'ancienne base électorale de Morales lui-même ${ }^{21}$. En effet, le peuple est devenu le principal contre-pouvoir à la politique d'Evo Morales, avec notamment les marches ou les blocus. Par ailleurs, l'usage des différents mécanismes de démocratie participative a été détourné de son sens premier, avec une utilisation davantage plébiscitaire que démocratique ${ }^{22}$. Le peuple a été écarté

17. Article 172 de la CPE de 2009.

18. Article 20 de la Constitution française du 4 octobre 1958.

19. Articles 159 et 160 de la CPE de 2009.

20. En la matière, le congrès bolivien est une institution très puissante dans le constitutionnalisme bolivien. En contribuant notamment à l'élection du Président de la République dans les constitutions antérieures à 2009, il s'était institué comme le principal contre-pouvoir du Président, dans le cadre de la « démocratie pactée », ou du " présidentialisme parlementarisé ». La CPE de 2009 a affaibli l'ALP comme contre-pouvoir, en la transformant en simple « chambre d'enregistrement ».

21. Dans les affaires du "Tipnis » ou du " gazolino » notamment, les peuples indigènes des basses-terres se sont opposés aux décisions du gouvernement d'Evo Morales et ses alliés cocaleros.

22. À ce sujet, le référendum de février 2016 sur la réforme constitutionnelle, qui a été portée par la Conalcam (Coordinadora Nacional para el Cambio), qui rassemble des mouvements sociaux alliés au MAS. 
des mécanismes démocratiques à l'origine prévus par la Constitution, et a été remplacé par les organisations alliées au MAS et à Morales ${ }^{23}$.

\section{2 - Le scrutin majoritaire à deux tours, un puissant facteur de personnalisation de la vie politique bolivienne}

De ce fait, la Constitution bolivienne opère un basculement inédit dans l'histoire constitutionnelle bolivienne. Le cas de l'élection du Président de l'État est, en ce sens, éloquent. Avant la promulgation de la CPE de 2009, l'élection du Président s'effectuait au scrutin majoritaire à un seul tour. Si aucun des candidats n'avait la majorité des voix, le Congrès national se réunissait et désignait le Président parmi les trois candidats arrivés en tête. Ce processus donnait lieu à des combinaisons politiques complexes ${ }^{24}$, obligeant certes à un consensus parlementaire autour d'une personnalité politique, mais se déroulant de manière opaque et donc peu propice à la démocratie. Ainsi, en 1989, c'est le troisième candidat arrivé en tête de l'élection qui fut élu en la personne de Jaime Paz Zamora, alors qu'il était derrière Gonzalo Sánchez de Lozada et Hugo Banzer $^{25}$. Ce mode d'élection était une constante dans le constitutionnalisme bolivien, et permettait un contrôle étroit du législatif sur l'exécutif par la désignation du président par le Congrès National. Cependant, si le consensus était un objectif louable dans le débat démocratique, il fut paradoxalement source d'instabilité, car les coalitions partisanes créées ne survivaient pas dans la durée, et contribuaient à l'alliance de partis aux programmes parfois totalement opposés. Ainsi, toujours lors de l'élection présidentielle de 1989, Hugo Banzer, ancien dictateur de la Bolivie ${ }^{26}$, et Jaime Paz Zamora, ancien opposant révolutionnaire à la dictature de Banzer, ont pu trouver un accord afin que Paz Zamora soit élu Président de la République, avec les voix de l'ADN ${ }^{27}$ de Banzer.

Depuis l'élection d'Evo Morales, le fait majoritaire fait que le Président, à l'instar de la France, concentre l'essentiel des pouvoirs politiques. La concordance des étiquettes politiques entre les pouvoirs exécutif et législatif a réduit considérablement le rôle de contre-pouvoir de l'ALP avec la disparition des coalitions partisanes. La collaboration

23. «El bloque hegemónico no constituye un bloque partidario propiamente dicho, siendo más bien una agregación o coalición de partidos, organizaciones y movimientos sociales, ONG con diversas identidades y orientaciones políticas ». E. Brockmann Quiroga, « La nueva Constitución Política del Estado », Miradas sobre el Nuevo Texto Constitucional, La Paz, Instituto Internacional para la Democracia y la Asistencia Electoral (IDEA Internacional), 2010, p. 392.

24. De la même manière que les « combinazione » dans le régime parlementaire italien.

25. Avec la révision constitutionnelle de 1994, afin d'éviter que ce scénario se reproduise, seuls les deux candidats arrivés en tête pouvaient prétendre à la désignation par le Congrès.

26. Hugo Banzer pris le pouvoir par un coup d'État militaire en 1971 et conserva le pouvoir jusqu'en 1978.

27. «Acción Democrática Nacionalista », Action démocratique nationaliste en français. 
devient ainsi subordination, et l'équilibre des pouvoirs n'est plus respecté. Aujourd'hui, la vie politique bolivienne se structure autour de l'élection présidentielle, qui avec son scrutin majoritaire à deux tours ${ }^{28}$ permet certes de dégager une «majorité présidentielle » claire, mais personnalise à l'extrême la vie politique bolivienne.

La question de la réélection présidentielle est prégnante dans le constitutionnalisme latino-américain, en partie à cause du régime présidentiel dominant. Aussi, la tentation est grande pour certains exécutifs de réintroduire dans les textes constitutionnels la possibilité d'une réélection présidentielle immédiate, comme au Venezuela, en Équateur, mais aussi en Bolivie. Alors qu'Evo Morales est à ce jour le Président bolivien avec le mandat le plus long de l'histoire du pays ${ }^{29}$, on constate un risque « d'hypertrophie présidentielle» avec une concentration du pouvoir politique comme une caractéristique du « nouveau constitutionnalisme bolivien ».

\section{II - LE « CONTINUISME » DES MANDATS PRÉSIDENTIELS : UNE MISE AU PAS DES DIFFÉRENTS ORGANES POLITIQUES AU SERVICE DU POUVOIR EXÉCUTIF}

La question de la réélection immédiate dans le droit constitutionnel bolivien, et notamment dans la jurisprudence du Tribunal Constitutionnel Plurinational (TCP). Après que le TCP ait interprété de manière très controversée la Disposition Transitoire Première ${ }^{30}$ de la CPE de 2009 (A), le pouvoir exécutif a voulu modifier l'article 168 de cette même constitution $^{31}$ afin de pouvoir se représenter à un quatrième mandat en 2019 (B).

\section{A - LA DISPOSITION TRANSITOIRE PREMIÈRE ET LE TRIBUNAL CONSTITUTIONNEL PLURINATIONAL : LE JUGE CONSTITUTIONNEL AU SERVICE DE L'ORGANE EXÉCUTIF}

Après avoir distingué au sein de la Constitution de 2009 une partie « dogmatique » et une partie « organique » (1), le juge constitutionnel a défini de manière pour le moins originale le « pouvoir constituant dérivé », accordant du même coup à l'ALP un certain pouvoir constituant (2).

28. Article 166 de la CPE de 2009.

29. Avant lui, seul le maréchal Andrés de Santa-Cruz était resté dix ans au pouvoir (1829-1839).

30. Tribunal Constitutionnel Plurinational, Déclaration Constitutionnelle Plurinationale $\mathrm{n}^{\circ} 0003 / 2013 \mathrm{du} 15$ avril 2013, Sucre.

31. L'article 168 de la CPE de 2009 dispose que « la période du mandat de la Présidente ou du Président et de la Vice-présidente ou du vice-président de l'État est de cinq ans, et ils peuvent être réélus une seule fois de manière continue ». 


\section{1 - La distinction entre partie dogmatique et partie organique dans la CPE de 2009}

En perspective de l'élection présidentielle du 12 octobre 2014², l'exécutif bolivien a commencé à remettre en question le principe de non-réélection immédiate. Un avant-projet de «loi normative d'application $^{33}$ » fut élaboré à la Chambre des sénateurs, afin de déterminer « l'application normative » de certaines dispositions constitutionnelles, notamment l'article 168 et la Disposition Transitoire Première de la CPE de 2009, qui se réferent tous deux à la réélection présidentielle. Ce projet de loi, contrairement à la loi $\mathrm{n}^{\circ} 4021$, considère que le premier mandat de l'actuel Président n'était pas celui qui était à l'œuvre lors de la promulgation de la CPE - c'est-à-dire entre 2006 et 2009 -, mais que le premier mandat était celui qui avait débuté en 2009. Afin de prévenir la probable opposition de droite qui voyait voler en éclat son accord politique, la Chambre des sénateurs a remis l'avant-projet de loi au Tribunal Constitutionnel Plurinational (TCP) en vue d'un contrôle de constitutionnalité.

Le TCP, dans l'article III.2 de la Déclaration Constitutionnelle Plurinationale $\mathrm{n}^{\circ} 0003 / 2013$ du 15 avril 2013, opère une distinction qualitative de la Constitution, entre une «partie dogmatique », et une «partie organique ». La partie dogmatique serait d'application directe dans la législation nationale, tandis que la partie organique aurait une applicabilité indirecte et conditionnée au nécessaire vote d'une «loi normative » :

«Dans le contexte signalé auparavant, il doit être précisé que dans tout État constitutionnel de droit, qui est une caractéristique de l'État plurinational de Bolivie, la partie dogmatique de la Constitution se caractérise par son application directe, c'est-à-dire que sa matérialisation et donc le phénomène de constitutionnalisation dans l'ordre juridique ne nécessitent pas de loi de développement ; au contraire, à la lumière du principe de légalité qui constitue un des piliers pour l'exercice de la fonction publique et au principe de sécurité et de continuité juridiques comme axes essentiels de l'État constitutionnel de droit, la partie organique de la Constitution, pour son application, nécessite des lois organiques de développement, lesquelles doivent émaner de l'ALP [...]. »

32. Les élections générales du 12 octobre 2014, où se déroulait l'élection présidentielle, mais également les élections législatives et sénatoriales, furent marquées par la victoire écrasante du MAS dans les deux chambres de l'ALP. Le parti d'Evo Morales remporta 88 des 130 sièges de la Chambre des députés, ainsi que 25 sièges sur 36 dans la Chambre des sénateurs. Evo Morales et Álvaro García Linera furent ainsi réélus dès le premier tour de l'élection présidentielle, avec $61,36 \%$ des suffrages exprimés.

33. Ce type de législation est l'équivalent des lois organiques dans le droit constitutionnel français. 
Cet argument rentre en contradiction avec la hiérarchie des normes établie par Hans Kelsen ${ }^{34}$, et la suprématie de la norme constitutionnelle dans la hiérarchie des normes. Loin du juge aiguilleur souhaité par Kelsen, on assiste plutôt à une véritable déformation du pouvoir d'interprétation du juge constitutionnel, ce qui pourrait faire naître des interrogations légitimes quant à un éventuel " gouvernement des juges ${ }^{35}$ » en Bolivie. Cependant, nous préférons nous appuyer ici sur la théorie du « réalisme interprétatif » de Michel Troper, qui considère le juge constituant comme un «co-législateur » à part entière ${ }^{36}$, aux côtés de l'organe législatif « classique ».

\section{2 - L’Assemblée législative plurinationale, un « pouvoir constituant dérivé »?}

Le TCP argue également de la nécessaire cohésion et contextualisation de la CPE par l'organe législatif. Le pouvoir législatif se concevrait comme un "pouvoir constituant dérivé », car l'organe représentatif du peuple souverain devrait pouvoir dépasser les contradictions apparentes de la CPE. La loi devient donc un outil de développement et de dépassement des supposées contradictions du pouvoir constituant originaire. Ainsi, s'il y a des contradictions dans la Constitution, ce n'est pas au TCP de les déterminer ni de les interpréter, mais au pouvoir législatif qui est alors considéré comme un pouvoir constituant dérivé. Il y a ici un renversement brusque - pour ne pas dire brutal - de la hiérarchie des normes, avec une subordination de la CPE à la loi ${ }^{37}$. Laffirmation selon laquelle l'ALP serait dépositaire d'un pouvoir constituant dérivé est un non-sens selon Alipio Valencia Vaga :

«Le pouvoir constituant est celui qui organise, structure réellement l'État [...] De l'autre côté, le pouvoir constitué réside dans l'ensemble des organes et des institutions établis par la Constitution et qui exercent les fonctions ou les activités du pouvoir public ordinaire, au quotidien [...] De cette manière, le pouvoir législatif ordinaire ne peut être un pouvoir constituant ;

34. H. Kelsen, Théorie pure du droit, Paris, LGDJ, « La pensée juridique », 1999.

35. E. Lambert, Le Gouvernement des juges et la lutte contre la législation sociale aux États-Unis, Paris, Giard, 1921, rééd. préf. F. Moderne, Paris, Dalloz, 2005.

36. Voir à ce sujet : M. Troper, Pour une théorie juridique de l'État, Paris, Presses universitaires de France, «Léviathan », $1^{\text {re }}$ édition, 1994.

37. La CPE, pour autant, n'a établi aucune compétence au législateur pour sanctionner des lois d'application. L'article 158.1 fait seulement mention que le législateur peut interpréter, voter, modifier, abroger des lois, mais pas des normes constitutionnelles. L'article 140.1: « Ni l'ALP ni aucun autre organe ou institution, association ou réunion populaire ne pourraient concéder à un organe ou une personne quelconque des facultés extraordinaires différentes à celles établies dans cette Constitution. "Dans cette décision, le TCP, de manière ironique, délègue des pouvoirs constituants à un organe législatif, alors qu'il est lui-même en charge de la défense et de l'intégrité de la Constitution. 
il est simplement un pouvoir constitué, et ne peut ainsi substituer ou modifier la Constitution, sinon en conformité avec les procédures que la Constitution a établies dans ces cas ${ }^{38}$. »

Pour justifier cette interprétation controversée de la Constitution, le TCP va arguer d'une nouvelle ère juridique en Bolivie : « Le nouvel ordre constitutionnel porté est différent de ce qui préexistait, puisque la fonction constituante, de par sa nature juridique, a généré une nouvelle ère juridicopolitique basée sur la refondation de l'État ${ }^{39}$. » Ainsi, la nature originelle de l'Assemblée constituante a amené une coupure dans la continuité juridicopolitique de la Bolivie, contrairement au principe de continuité qui est l'un des fondements de l'État de droit. C'est l'argument du pouvoir constituant originaire qui légitime une « coupure ", un avant et un après dans le système juridique bolivien ; bref, un «néant juridique » avant 2009. Mais si l'argument du pouvoir constituant originaire s'écroule, c'est toute l'argumentation du TCP qui s'effondre du même coup ${ }^{40}$.

De ce fait, le TCP a nié la volonté du constituant, qui était de limiter le nombre de réélections possibles du mandat présidentiel. Et bien que la Disposition Transitoire Première fût introduite suite à un accord politique négocié par le Congrès National, la volonté du constituant était bien de limiter le nombre de mandats présidentiels, au travers de l'article 168 de la CPE de 2009. Or, bien que l'objet de la décision soit la Disposition Transitoire Première, il n'en reste pas moins que l'article 168 limite à une seule réélection le mandat présidentiel. En donnant une interprétation de la Disposition Transitoire Première contraire à elle-même, mais également contraire à l'article 168, le TCP rend une décision intrinsèquement contradictoire : le TCP affirme que le rôle du supposé pouvoir constituant dérivé de l'ALP serait de « mettre de l'ordre » dans la Constitution; or, il provoque lui-même ce désordre.

\section{B - UNE LOI DE RÉVISION CONSTITUTIONNELLE «ANTICONSTITUTIONNELLE »}

Evo Morales réélu triomphalement à l'élection présidentielle du 12 octobre 2014, la question d'une nouvelle réélection se posait, cette fois-ci pour l'échéance de 2019. L'interprétation très « extensive » du

38. A. Valencia Vaga, Teoria politica, tome II, La Paz, Juventud, 2édition, 1978, p. 236. 39. Tribunal Constitutionnel Plurinational, Déclaration Constitutionnelle Plurinationale $\mathrm{n}^{\circ} 0003 / 2013,25$ avril 2013 , Sucre.

40. Selon Kelsen, la seule possibilité pour qu'il y ait une coupure et qu'il n'y ait pas de continuité entre deux textes constitutionnels a lieu lorsque le nouveau texte est promulgué hors des procédures établis par son prédécesseur. Or, l'Assemblée constituante de 2006 procède de la «Ley especial de convocatoria a la asamblea constituyente » du 6 mars 2006, qui se base elle-même sur la « Ley Especial 3091 » du 6 juillet 2005. 
TCP étant allée jusqu'à ses limites, il est cette fois nécessaire de modifier le fameux article 168 de la CPE de 2009 afin de permettre au Président actuel de se représenter en 2019. La tentative de modifier l'article 168 s'est pourtant révélée aussi bien inconstitutionnelle sur la forme (1) que sur le fond (2).

\section{1 - Une procédure de révision inconstitutionnelle sur la forme}

Parmi les 411 articles qui composent la CPE de 2009, un seul traite de la révision - partielle ou totale - du texte constitutionnel : l'article 411. La révision partielle nécessite le vote d'une Loi de réforme constitutionnelle, qui doit être approuvée aux deux tiers des voix de l'ALP ${ }^{41}$. Ainsi, pouvoir constitué et pouvoir constituant sont étroitement liés au processus de révision, le pouvoir constitué prenant effectivement la décision d'entreprendre une procédure de révision, tandis que le pouvoir constituant approuve ou rejette in fine la révision constitutionnelle. En ce sens, une loi de révision constitutionnelle fut présentée à l'ALP le 16 septembre 2015, afin de modifier l'article 168 et permettre une quatrième réélection présidentielle d'Evo Morales en 2019. Selon Carlos Andrés Miranda-Ramirez ${ }^{42}$, cette loi est foncièrement anticonstitutionnelle et contredit à de nombreuses reprises la CPE de 2009. Tout d'abord, celle loi présente un vice de forme, car la procédure législative ad hoc n'a pas été respectée. On peut aussi dégager un vice de compétence, car cette révision partielle de la Constitution, conformément à l'article 411.1 de la $\mathrm{CPE}$, ne constitue pas une révision de « deuxième degré » - c'est-àdire une révision partielle de la Constitution - mais bien de «premier degré »- une révision totale de la Constitution. Dans ce sens, une révision de l'article 168 aurait nécessité la convocation d'une Assemblée constituante, chose qui n'a pas été faite pour la présente réforme ${ }^{43}$.

41. On trouve tout d'abord, une phase d'initiative, qu'elle soit d'origine populaire ou législative, ou judiciaire, ou exécutive. Ensuite, la phase de discussion et d'approbation par l'ALP. La proposition de loi, une fois arrivée dans la première chambre d'examen, est envoyée à la commission ad hoc, puis soumise au débat et au vote (à la majorité simple). La proposition de loi est ensuite envoyée à l'autre chambre, et examinée dans les mêmes conditions. La loi est par la suite votée à la majorité qualifiée cette fois par l'ALP dans son ensemble (article 411.II). La loi est envoyée à l'organe exécutif qui la promulgue. Enfin, la loi de révision partielle est approuvée par référendum. Ce dernier ne tire pas sa genèse de la loi de révision, mais d'une autre loi appelée «Loi de convocation au référendum », la question étant examinée par le Tribunal Suprême Electoral puis soumise au contrôle de constitutionnalité par le TCP.

42. C.-M. Miranda-Ramírez, « La inconstitucionalidad ternaria de la Ley de Reforma parcial de la Constitución. Lo imprejuzgado en la DCP 0193/2015 », Santa Cruz, Seminario nacional sobre la reelección presidencial, Academia Boliviana de Estudios Constitucionales, novembre 2015 .

43. Ce qui a d'ailleurs été reconnu à demi-mot par le TCP dans sa décision 0193/2015 du 21 octobre 2015 . 
C'est la Conalcam ${ }^{44}$ qui fut à l'origine de cette révision constitutionnelle, à travers une initiative législative citoyenne ${ }^{45}$ qui fut transmise au Président de l'ALP, José Alberto Gonzales Samaniego. Ce même 17 septembre 2015, ce qui était une initiative citoyenne, et qui nécessitait par la même occasion la procédure législative doit être portée devant la Chambre des députés - est étrangement devenue une initiative législative des assembléistes ${ }^{46}$. Enfin, toujours le même jour, le Président de l'ALP a remis ce qui était désormais un projet de loi de révision partielle à la Présidence de la Chambre des Sénateurs. Le 18 septembre, la procédure législative du projet de loi de révision partielle a débuté. Cependant, le Président de la Chambre des sénateurs doit remettre le projet de loi à la Commission mixte de la Constitution, des droits humains, de la législation et du système électoral de l'ALP, alors que conformément à l'article 411.2 de la CPE, la chambre qui a déposé ce projet de loi doit le voter avant que le projet puisse être approuvé par l'autre chambre, pour finalement être voté en séance plénière par l'ALP aux deux tiers des membres présents. On constate donc que la procédure suivie pour la révision partielle de la Constitution ne respecte pas la procédure indiquée dans cette même Constitution ; il y a une inconstitutionnalité sur la forme. Par ailleurs, alors même que le texte n'était qu'un projet de loi, ce dernier fut soumis au TCP pour un contrôle de constitutionnalité, ce qui était contraire à l'article 202.10 de la CPE, et aux articles 149 et 152 du Code de procédure constitutionnelle.

Selon l'article 162.I de la CPE de 2009, une révision partielle de la Constitution peut être décidée à partir d'une initiative législative sous la forme d'une proposition de loi, qui peut être présentée devant l'ALP par les citoyens, les assembléistes, l'organe exécutif, le TSJ, et les gouvernements autonomes des entités territoriales. Cependant, la CPE de 2009 ne précise pas quel type de procédure législative doit être engagé pour cette proposition de loi ; tout au plus elle nous renseigne sur le vote d'une loi ordinaire, mais non sur une loi de révision constitutionnelle. Devant le silence de la Constitution de 2009, Carlos Andrés MirandaRamirez préfere se référer à l'article 230 de la Constitution de 1967, selon lequel la révision partielle peut être effectuée avec une loi ordinaire approuvée aux deux tiers des membres présents dans chacune des chambres. Cette loi «déclaratoire » sera ensuite promulguée par l'exécutif. L'article 231 de la Constitution de 1967 précise qu'une loi de réforme sera ensuite votée aux deux tiers dans chacune des chambres, de la même manière et dans les mêmes termes, dans le cadre de la loi

44. «Coordinora nacional para el cambio », organisation qui rassemble les organisations sociales alliées au MAS.

45. Article 163.1 de la CPE de 2009.

46. «Une mutation arbitraire d'un acte juridique » selon C. A. Miranda-Ramirez. 
déclaratoire. La loi de réforme sera ensuite sanctionnée par l'exécutif, avant sa promulgation par ce dernier ${ }^{47}$. De cette manière, Carlos Andrés Miranda-Ramirez en déduit que la procédure à engager pour une révision partielle devrait être celle d'une procédure législative ordinaire, actuellement régie à l'article 163 de la CPE de 2009, avec cependant deux conditions : l'approbation de la loi doit s'effectuer à la majorité qualifiée des deux tiers (et non à la majorité simple), et elle doit ensuite être soumise à un référendum.

Nous allons maintenant voir dans quelle mesure la décision du TCP constitue, en outre, une inconstitutionnalité sur le fond.

\section{2 - Une loi de révision anticonstitutionnelle sur le fond}

La loi de révision n'est matériellement pas conforme à la Constitution, car en remettant en cause la limitation des mandats présidentiels, cette révision fragiliserait les « bases fondamentales » de la démocratie, à savoir le principe de séparation des pouvoirs, et surtout le principe d'alternance du pouvoir politique, qui est une des conditions principales de l'exercice de la démocratie représentative ${ }^{48}$.

Dans la décision DCP 0193/2015 du 21 octobre 2015, le TCP définit notamment ce qui relève de la révision totale de la Constitution - ce qui affecte les bases fondamentales de la Constitution - et la révision partielle - qui ne peut par «effet de miroir » concerné que les objets superficiels et mineurs d'une constitution. Selon le juge constitutionnel, la loi de révision partielle "n'affecte pas la démocratie ni les principes constitutionnels, étant donné que la proposition est claire et catégorique

47. L'ancien Tribunal Constitutionnel précisait, à travers sa décision «AC 568/2006-CA » du 7 novembre 2006, dans sa partie III.5 : «En el caso de la reforma parcial, el procedimiento se inicia con la Ley de Declaratoria de Necesidad de Reforma de la Constitución, que podrá tener su origen en cualquiera de las Cámaras, para luego ser analizado, discutido y aprobado en grande, en detalle y en revisión, conforme al procedimiento legislativo previsto por los arts. 71 al 75 de la CPE, para que una vez sancionada, se remita al Poder Ejecutivo para la respectiva promulgación, sin derecho a veto. Posteriormente, de conformidad a lo previsto por el art. 231 de la Ley Fundamental, en las primeras sesiones de la legislatura de un nuevo período constitucional, en la Cámara de origen se analizará y aprobará la Ley de Reforma de la Constitución, la que contendrá la disposición expresa de reformarla, enumerando los respectivos artículos objeto de la reforma y con el que modifican dichos artículos. La referida norma se aprueba con la votación cualificada de dos tercios de los miembros presentes en cada una de las Cámaras, sujetando los trámites al procedimiento legislativo establecido por los arts. 71 al 75 de la CPE, y una vez sancionada la Ley, será remitida al Poder Ejecutivo para su respectiva promulgación, sin lugar a veto. »

48. Cette question fondamentale de la compétence renvoie à une problématique qui touche l'ensemble des textes constitutionnels nouvellement approuvés : comment appliquer, dans le respect de la hiérarchie des normes, les dispositions constitutionnelles qui nécessitent un « développement »? Bien souvent, ces dispositions indiquent la procédure adéquate pour être développées. La Constitution française s'est mise en place grâce aux ordonnances du gouvernement, mais surtout grâce aux lois organiques qui nécessitent une procédure législative plus stricte pour être approuvées. 
“pour deux fois consécutives”, c'est-à-dire [qu'elle a] une limite constitutionnelle ». Le TCP se range derrière son rôle de contrôle unique de la forme, en affirmant que « par mandat constitutionnel et légal [le TCP ] se limite à vérifier uniquement la constitutionnalité ou non de la procédure et de la Loi de révision constitutionnelle ». Surtout, le TCP estime que la révision de l'article 168 , avec la remise en cause du principe de réélection présidentielle unique, ne fait pas partie d'une révision totale, mais bien d'une révision partielle.

Arrêtons-nous un instant sur l'interprétation faite par le juge constitutionnel de l'article 168, car elle est déterminante dans la poursuite de notre réflexion. Selon le TCP, l'article 168 présenterait deux « faces juridiques ». La première face serait de nature organique en ce qu'elle détermine la durée d'un mandat présidentiel. La seconde face, serait quant à elle «axiologique », ou «dogmatique» pour reprendre le vocable juridique du TCP : elle limite un mandat présidentiel à une seule réélection continue, ce qui détermine la forme démocratique du gouvernement de Bolivie ${ }^{49}$. L'article 168, bien qu'il se trouve dans la partie organique de la $\mathrm{CPE}$, présente dans sa composition une facette « axiologique » en ce qu'il établit une « limite axiologique à l'exercice du pouvoir politique de l'organe exécutif, en interdisant de manière explicite une seconde réélection consécutive ${ }^{50} »$. Selon Carlos Andrés Miranda-Ramírez, l'article 168 n'a d'autre but que de «préserver l'idéologie démocratique du nouvel État bolivien (du Préambule jusqu'à la disposition finale de la Constitution) et le plein respect de l'État constitutionnel et démocratique de droit plurinational en Bolivie, de sa forme de gouvernement républicain (où un mandat fixe et une réélection consécutive limitée constituent les bases fondamentales) et des droits fondamentaux (l'égalité et les droits politiques) au niveau du vote des lois et des processus électoraux pour le respect de l'État constitutionnel ainsi que de l'État bolivien ${ }^{51} »$. À partir de ce développement, l'auteur en vient à conclure que cette révision partielle était marquée du sceau de l'inconstitutionnalité, et que par conséquent le référendum du 21 février 2016 qui en découlait était lui aussi anticonstitutionnel.

49. Le TCP, dans le fondement juridique III.2 de la Déclaration Constitutionnelle Plurinationale $0003 / 2013$ du 25 avril 2013, dispose que « la partie dogmatique de la constitution dispose des valeurs suprêmes, des principes recteurs, des droits fondamentaux et des garanties normatives, juridictionnelles et de défense. De même, la partie organique de la Constitution structure comme il a été dit précédemment l'ingénierie institutionnelle (sic) qui dans l'État Plurinational de Bolivie, devra répondre au pluralisme, à l'interculturalité et aux postulats propres à l'État constitutionnel de droit. Ainsi, en suivant les axes directeurs établis dans la partie dogmatique de la constitution, la partie organique traduit l'organisation de l'exercice du pouvoir destiné essentiellement à réaliser les fins essentielles de l'État plurinational de Bolivie. »

50. C. A. Miranda-Ramírez, op. cit., p. 26.

51. Ibid., p. 26. 
On peut résumer les choses ainsi. Tout d'abord, la loi n'a pas suivi la procédure législative prévue dans l'article 163 et l'article 411.II. En opposition à la loi, l'initiative citoyenne soumise par la Conalcam ne comportait même pas la signature de $20 \%$ des électeurs, et s'est transformée de manière illégale en initiative législative de la seule volonté du groupe MAS de l'ALP. Le vendredi 18 septembre 2015, le Président de l'ALP a remis la proposition à la chambre des sénateurs, au lieu de la chambre des députés. Par la suite, la proposition a été soumise à la commission mixte sur la Constitution, les droits humains, la législation et le système électoral de l'ALP, alors que normalement la commission de la chambre des députés aurait dû l'examiner en première. On constate ainsi que la procédure législative est totalement éloignée de la procédure prévue à l'article 163 de la CPE de 2009. Enfin, on constate également une inconstitutionnalité sur le fond : cette réforme engagée par l'ALP portait sur un élément « axiologique » de l'article 168, c'est-à-dire portant sur des principes fondamentaux et intangibles de la Constitution, qui ne peuvent être modifiés que par une révision totale de la Constitution, ce qui implique une procédure autrement plus lourde et complexe à mettre en ouvre, avec notamment la convocation d'une Assemblée constituante.

Ce qui distingue le constitutionnalisme latino-américain, et en particulier le constitutionnalisme bolivien, c'est la fragilité et la vulnérabilité des pouvoirs législatifs et judiciaires face au pouvoir exécutif et surtout face à la fonction présidentielle. On pourrait même concevoir un lien entre la fragilité des institutions et la tendance au caudillisme. Jorge Lazarte affirme à ce titre que le « caudillisme est l'expression la plus forte de la précarité des institutions, et il existe entre ces deux termes une relation inversement proportionnelle ${ }^{52} »$. Le constitutionnaliste José Antonio Rivera Santiváñez, pourtant favorable au « proceso de cambio », vient préciser cette théorie. Selon lui, la principale menace de ce nouveau constitutionnalisme est de générer un phénomène de "déconstitutionnalisation » caractérisé par un manque de loyauté envers la Constitution de la part des gouvernants ${ }^{53}$. Ainsi, l'État ne respecte pas la volonté du constituant - et donc en partie la sienne - en ne mettant pas en œuvre les dispositions législatives et réglementaires pour rendre effectifs les droits inscrits, et en prenant des décisions en contradiction avec la Constitution. En ce sens, les décisions répétées du juge constitutionnel bolivien au sujet

52. J. Lazarte, «Estado plurinacional y Estado de derecho. ¿Dos modelos políticos democráticos? ", Realidad y perspectivas del constitucionalismo boliviano: memorias del Segundo congreso boliviano de derecho constitucional, Cochabamba, Grupo Editorial Kipus, Academia Boliviana de Estudios Constitucionales, 2014.

53. J. A. Rivera Santiváñez, "Avances, riesgos y desafíos del constitucionalismo latinoamericano ", Realidad y perspectivas del constitucionalismo boliviano: memorias del segundo congreso boliviano de derecho constitucional. Cochabamba, Grupo Editorial Kipus, Academia Boliviana de estudios constitucionales, 2014. 
de la possible réélection du «ticket » Morales/Garcia Linera confirme le fait que le TCP est un organe subordonné au pouvoir exécutif, remettant en cause certains principes fondamentaux de la CPE de 2009, telle que la limitation à deux mandats présidentiels successifs.

Le constituant bolivien de 2009 a cru bon de mettre un certain nombre de garde-fous à une révision - partielle ou totale - du texte constitutionnel. En particulier, la nécessité d'en appeler à un référendum constitue l'un des derniers remparts démocratiques face à la résurgence d'une certaine forme de pouvoir très personnel : le caudillisme. Les organisations alliées à Evo Morales réclament déjà un second référendum sur la modification de l'article 168, afin de lui permettre de conserver le pouvoir jusqu'en 2025, date anniversaire du bicentenaire de l'indépendance de la Bolivie. Le peuple bolivien, plus que jamais, est seul maître de son destin. 
